\title{
Do We Need More Than One ThinPrep to Obtain Adequate Cellularity in Fine Needle Aspiration?
}

\section{Dear Dr. Bedrossian:}

Here in, we report our study regarding the value of additional or minimal number of ThinPrep slides per case needed to establish a correct diagnosis on cytology specimens. The objective of this study is to explore whether preparing a second ThinPrep (TP) slide that presumably contains additional clusters would improve the diagnostic accuracy of fine needle aspiration (FNA)? To the best of our knowledge there have been no studies to establish the minimal number of TP slides on aspirated materials.

Over the last 10 years, TP an automated technique has been used to prepare slides not only for cervical-vaginal samples but also for a wide range of nongynecologic specimens including fine needle aspirates. It has been shown by several studies that TP has improved limitations previously experienced on conventional smears such as obscuring material (blood, exudate), difference in smear thickness, and air drying artifacts. ${ }^{1-7}$

In July 1995, the TP method was implemented at the University of Michigan for nongynecologic specimens. Only one TP slide has been prepared for each aspirate in our laboratory. While it is known that TP provides a random representative preparation of the obtained sample, it has not been established whether additional preps in low cellular specimens would provide additional information to establish a conclusive diagnosis. The literature suggests that in the Pap test, an additional TP slide carries the chance to detect a potential abnormality. ${ }^{8}$

The utilization of liquid based preparation (LBP) for FNA provides several advantages for both the laboratory and the clinicians performing those FNAs. In addition to the known advantages such as simpler collection, better

*Correspondence to: Farnaz Hasteh, M.D., 200 West Arbor Dr, Rm 2-120, San Diego, CA 92103-8720. E-mail: fhasteh@ucsd.edu Received 2 March 2007; Accepted 22 August 2007

DOI 10.1002/dc.20735

Published online in Wiley InterScience (www.interscience.wiley.com). preservation and elimination of obscuring elements, the concept is particularly attractive to large laboratory corporations with multiple centers throughout the nation as well as small private laboratories. The ability to reliably transport a specimen and to prepare a well preserved slide without investing on onsite assistance or technical support is an attractive principle to these institutions. Moreover, LBP shifts the control over the specimen to the laboratory.

In FNAs, such as thyroid and breast, cellularity and number of clusters are crucial to consider the specimen adequate. However, it has been our observation that even in low cellular sample, not all clusters are represented in one TP. Whether additional TP, in suboptimal samples would provide additional cells/clusters and assist in establishing a diagnosis is not well established. In a study by Frost et al., they concluded that 1.43 slides were adequate. $^{9}$

In the majority of our FNA biopsies, the conventional smears are prepared on site by pathologists or cytotechnologists. In theses cases, the needle is rinsed in CytoLyt solution after making the smears, and at least one pass is dedicated to make a TP slide. In contrast, when the clinicians perform the FNA in their clinic, they are instructed to rinse the needle directly in CytoLyt and send it to the laboratory. In this setting, a TP and a cell block are prepared.

The specimen is transferred to $50 \mathrm{ml}$ tube and centrifuged at $1500 \mathrm{rpm}$. Two to three drops of the cell pellet are transferred to PreservCyt and one TP slide is prepared by the Cytyc 2000 according to the manufacture's brochure. A cell block is prepared by the histogel method from the remaining cell pellet. In this study, a total of 100 consecutive FNAs were processed with 2 TP slides prepared for each case. FNAs were obtained from the following sites: thyroid (40 cases), breast (17 cases), salivary gland (10 cases), skin lesions (10 cases), head and neck lesions (eight cases), lymph node (eight cases), gastrointestinal tact (seven cases), and ovary (two cases). All cases were included after the final diagnosis was reported 
to the clinicians. In $40 / 100$ of cases, additional smears were available and in 60/100 of cases only TP slides were available. The comparison between the qualities of the direct smears vs. the TP slide was not made since this was not part of our study. Each TP slide was reviewed blindly and separately without knowledge of the final reported diagnosis by one cytopathologic (FH). A combined diagnosis was consequently rendered based on reviewing both TP slides together. Diagnostic categories are summarized in Table I.

The diagnosis on each TP slide was compared with each other and against the combined TP diagnosis and the final reported diagnosis.

In $97 \%$ of the cases, no additional information was obtained from the second TP slide. In suboptimal or nondiagnostic cases both TP slides showed similar patterns (low cellularity or acellular). Only in three cases, the second TP slide had rare atypical cells while the first slide

\section{Table I. Diagnostic Categories}

1. Insufficient for diagnosis due to the low cellularity.

2. Benign diagnosis.

3. Suspicious due to significant cellular atypia but not enough to make

the diagnosis of malignancy.

4. Malignant due to presence of definitive malignant cells.

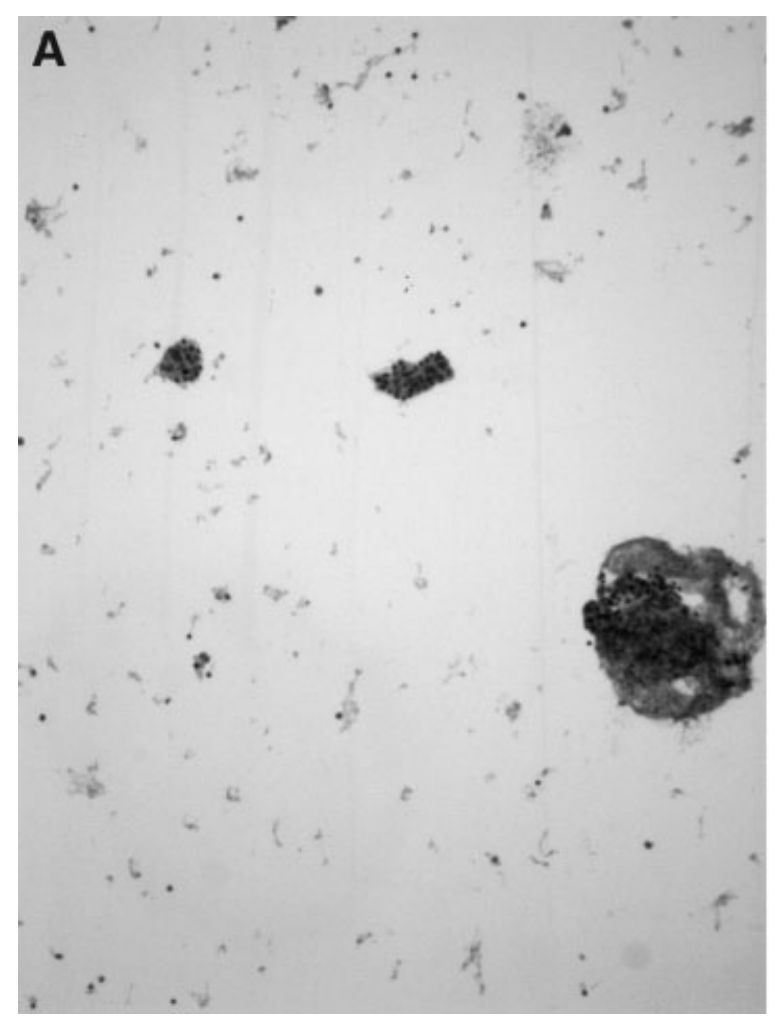

was acellular; however, the number of atypical cells was still not sufficient for a definitive diagnosis. In 8/40 cases, the final diagnosis was made based on the cytologic smears and not on TP slides. In all of the cases (100\%) the combined diagnosis based on the two TP slides was the same as the diagnostic category of the first TP slide (Figs. 1-3).

On the basis of our study, we believe that one TP slide per case is adequate to represent a specimen for a diagnosis. In $97 \%$ of cases, reproducibility between both TP slides was excellent with the second TP slide either equal or less cellular. Only 3\% had additional atypical cells on the second TP slide, yet not sufficient for diagnosis and would not justify the additional cost.

\section{Farnaz Hasteh, M.D.* \\ Department of Pathology \\ University of California \\ San Diego}

Yijun Pang, M.D., Ph.D.

Robert Pu, M.D., Ph.D.

Claire W. Michael, M.D.

Department of Pathology

University of Michigan Medical Center

Ann Arbor, Michigan

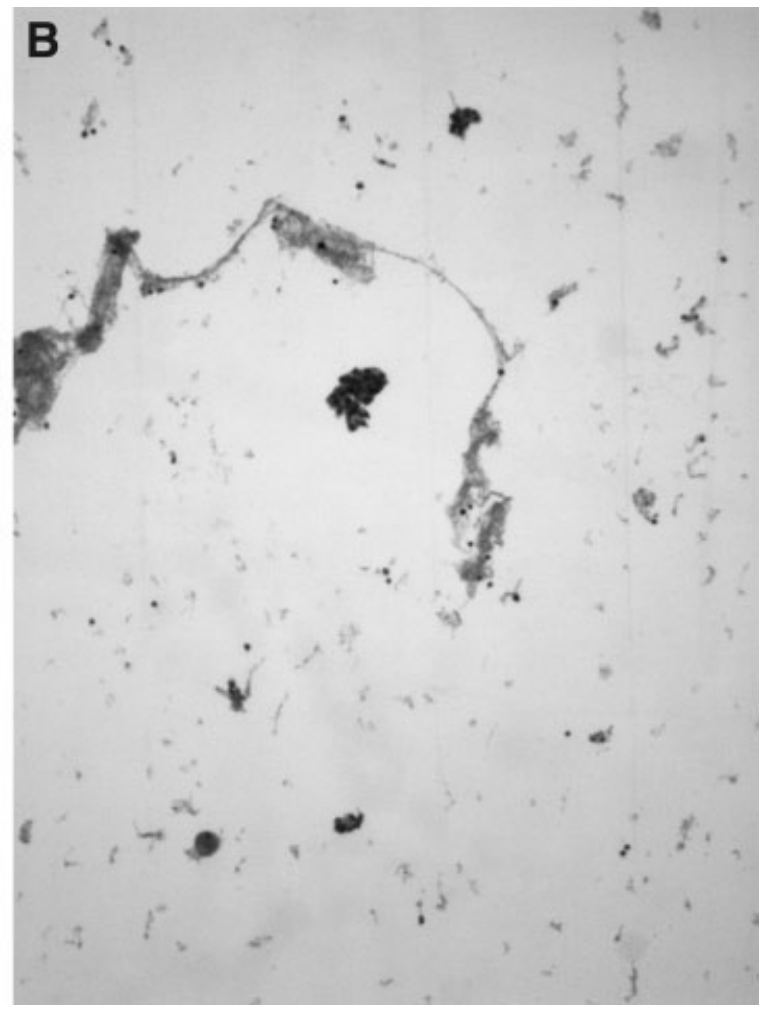

Fig. 1. A, B: FNA of thyroid. Low cellularity on both TP slides $(\times 100)$. Diagnosis: Insufficient for diagnosis due to low cellularity. Rare clusters of bland follicular cells and macrophages. 

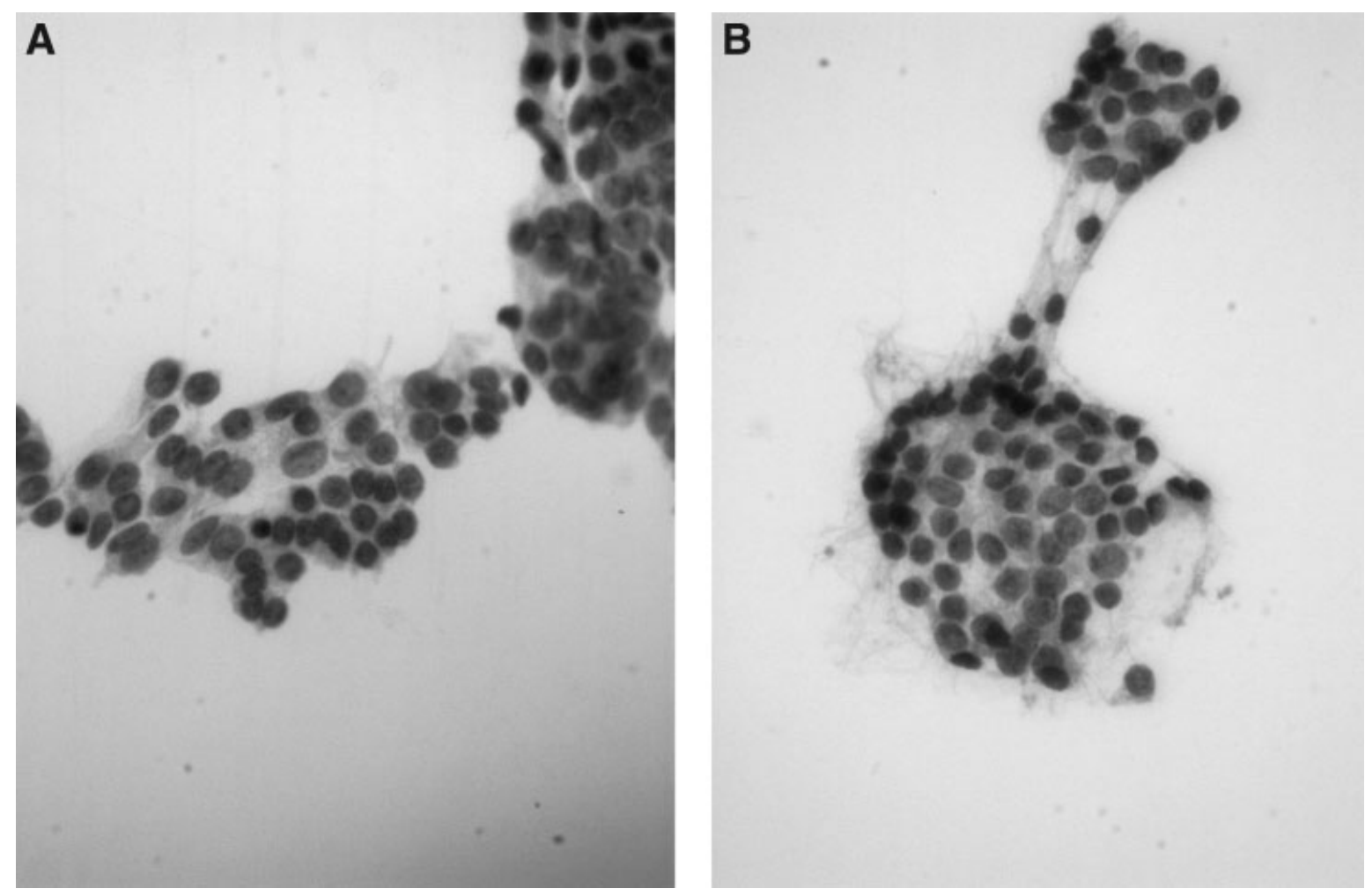

Fig. 2. A, B: FNA of thyroid. Moderate cellularity on both TP slides (not shown here). Similar groups of atypical follicular cells suspicious for papillary thyroid carcinoma $(\times 400)$. Surgical excision of the mass was papillary thyroid carcinoma.
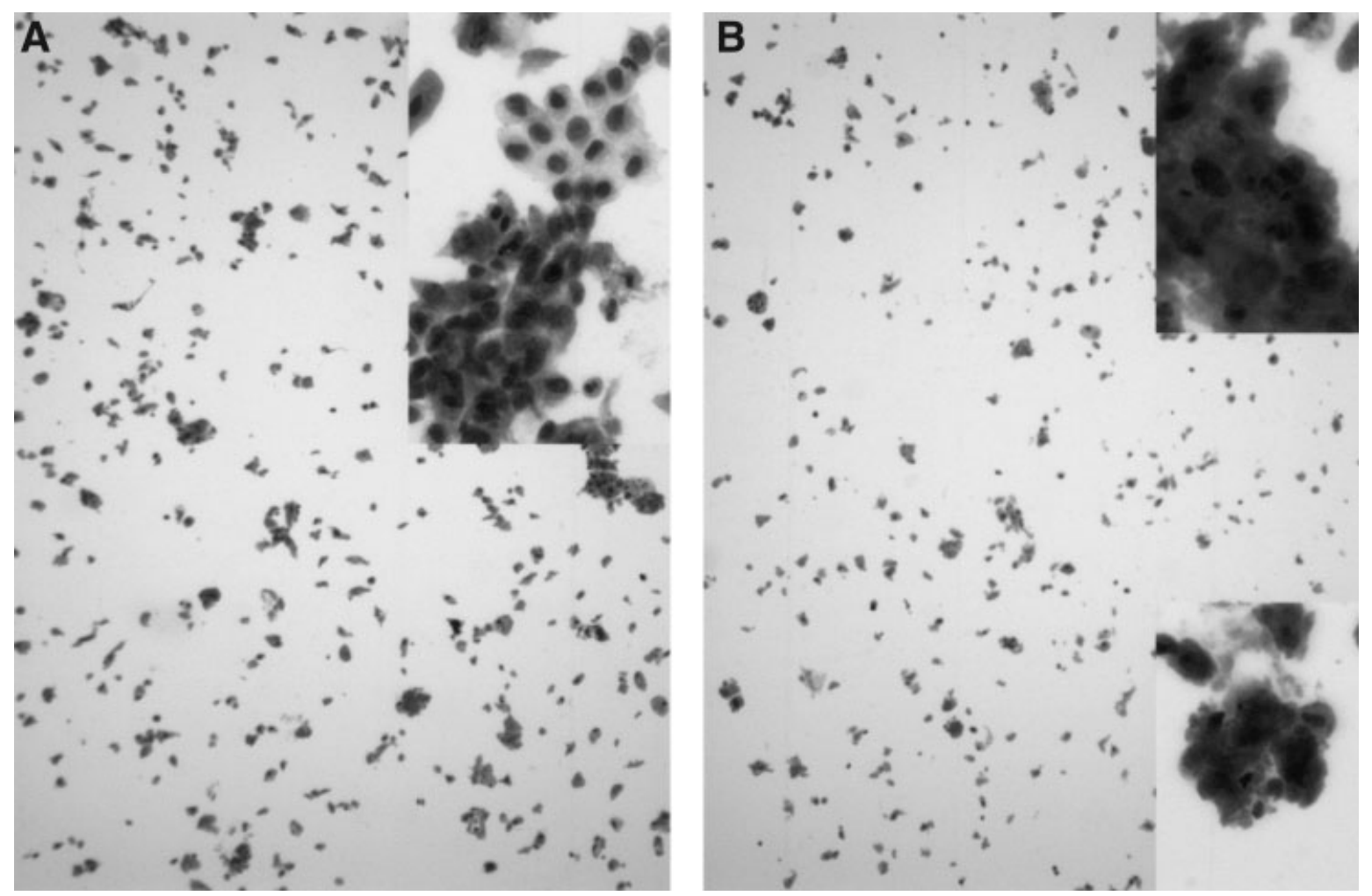

Fig. 3. A, B: Neck FNA, richly cellular on both TP slides. Similar diagnostic materials on both TP slides. Diagnosis: Metastatic squamous cell carcinoma. $(\times 100$ and $\times 400)$. 


\section{References}

1. Awen C, Hathways S, Eddy W, et al. Efficacy of ThinPrep preparation of cervical smears: A 1000 case, investigator-sponsored study. Diagn Cytopathol 1994;11:33-36.

2. Huchinson MI, Cassin CM, Ball HG. The efficacy of an automated preparation device for cervical cytology. Am J Clin Pathol 1991;96: 300-305.

3. Biscotti CV, Hollow JA, Toddy SM, et al. ThinPrep versus conventional smear cytologic preparations in the analysis of thyroid fine needle aspiration specimens. Am J Clin Pathol 1995;104:150 153.

4. Lee KR, Papillo JL, St. John T, et al. Evaluation of the ThinPrep processor for fine needle aspiration specimens. Acta Cytol 1996;40: 895-899.
5. Hees K, Lebeau PB. Comparison of conventional and ThinPrep preparations of mucoid cytology samples. Diagn Cytopathol 1995;12: 181-185.

6. Michael CW, Hunter B. Interpretation of fine needle aspirated processed by ThinPrep technique. Diagn Cytopathol 2000;23:6-13.

7. Thinprep 2000 operator's manual. Malborough, MA: Cytec Corporation; 1995.

8. Bentz JS, Rowe LR, Gopez EV, et al. The unsatisfactory ThinPrep Pap Test: Missed opportunity for disease detection? Am J Clin Pathol 2002;117:457-463.

9. Frost AR, Sidaway MK, Ferfelli M, et al. Utility of thin-layer preparations in thyroid fine needle aspiration. Diagnostic accuracy, cytomorphology and optimal sample preparation. Cancer Cytopathol 1998;84:17-25. 